\title{
SALUD MENTAL, CONFINAMIENTO Y PREOCUPACIÓN POR EL CORONAVIRUS: UN ESTUDIO CUALITATIVO
}

\author{
Gabriela Vásquez ${ }^{1}(\mathbb{D}$, Óscar Rolando Urtecho-Osorto $(\mathbb{D}$, Maitée \\ Agüero-Flores (D), Miguel José Díaz-Martínez (D), Rosa María Paguada \\ (D), Melody Anette Varela (D), Miguel Landa-Blanco (D), \& Yaraní \\ Echenique
}

Universidad Nacional Autónoma de Honduras, Tegucigalpa, Honduras.

\begin{abstract}
RESUMEN
Este estudio cualitativo explora las preocupaciones vinculadas con la COVID-19, el confinamiento y su relación con la salud mental en el contexto hondureño. Incluyó 20 entrevistas semiestructuradas realizadas a 10 hombres y 10 mujeres. Se halló que hay personas preocupadas por la salud propia y la de sus seres queridos. Esto puede detonar reacciones psicosomáticas y ansiedad. Otras personas devalúan la gravedad de la COVID-19 porque no se consideran vulnerables. Exponerse recurrentemente a información mediática resulta estresante, así que algunos evitan hacerlo. Otra preocupación es económica: reducción del acceso al mercado laboral y suspensión o disminución del ingreso. Algunos participantes experimentan alteraciones en los hábitos alimenticios y de sueño. Finalmente, aunque el confinamiento limita la recreación, los informantes han realizado actividades de ocio.
\end{abstract}

Palabras Claves

coronavirus; pandemias; salud mental; psicología en salud; cuarentena

\section{AbSTRaCT}

This qualitative study explores concerns related to COVID-19, confinement, and its relationship to mental health in the Honduran context. 20 semi-structured interviews to 10 men and 10 women were included. It was found that there are people concerned about their own health and their loved ones'. This can trigger psychosomatic reactions and anxiety. Other people underestimate the graveness of COVID-19 because they don't consider themselves vulnerable. To be recurrently exposed to media information is stressful, so some people avoid it. Economic concern is also relevant due to reduced access to labor market and the suspension or decrease of income. Some informants are experiencing alterations in their eating and sleeping habits. Lastly, even though confinement limits leisure events, the informants have engaged in recreational activities.

\section{Keywords}

coronavirus; pandemics; mental health; behavioral medicine; quarantine

\footnotetext{
${ }^{1}$ Correspondence about this article should be addressed to Gabriela Vásquez: gvasquezg@unah.hn
} 


\section{MENTAL HEALTH, CONFINEMENT, AND CORONAVIRUS CONCERNS: A QUALITATIVE STUDY}

\section{Introducción}

La enfermedad COVID-19 es causada por el SARS-CoV-2. Virus de este tipo, al ser trasmitidos de animales a humanos, en años anteriores dieron origen a las epidemias del Síndrome Respiratorio Agudo Severo y el Síndrome Respiratorio del Medio Oriente (Shereen et al., 2020). El primer diagnóstico de este nuevo coronavirus se realizó a finales de diciembre de 2019 en Wuhan, China. El virus se propagó rápidamente y el primer caso fuera de China fue identificado en enero de 2020 (Wu \& McGoogan, 2020).

Según datos tomados al 1 de junio de 2020, las cifras de la COVID-19 han aumentado hasta alcanzar los 6203385 contagios a nivel global. Esta cifra visibiliza el alcance mundial de la enfermedad. A esto hay que agregar que el número de muertos en todo el planeta llega a los 372657 (Johns Hopkins University and Medicine, 2020). Para esta misma fecha, Honduras reportaba 5202 casos confirmados y 212 decesos (Despacho de Comunicaciones y Estrategia Presidencial, 2020). Ante el creciente número de infectados, hay expertos que consideran que el sistema de salud hondureño tiene escasa capacidad para hacer frente a la pandemia e incluso podría colapsar. Esto se evidencia en la limitada aplicación de pruebas PCR para la detección de la COVID-19, la demora en el procesamiento de dichas pruebas, el escaso personal médico contratado y en la carencia de los recursos hospitalarios necesarios para asistir a la población (Sosa, 2020).

Por su letalidad, rápida propagación, consecuencias sociales y difusión mediática, se sabe que el virus, además de su impacto en el bienestar físico, puede afectar la salud mental de las personas (Rodríguez Cahill, 2020). La salud mental se caracteriza por el bienestar emocional, la adaptación a los cambios, la capacidad de establecer relaciones interpersonales sanas, el manejo adecuado del estrés del diario vivir y el carecer de padecimientos o trastornos psicológicos (American Psychological Association, 2020). Es importante mencionar que la presencia de síntomas psicológicos puede afectar varias áreas de la vida, como las relaciones interpersonales, las relaciones familiares, el desempeño laboral, las capacidades de aprendizaje y la participación en actividades cotidianas importantes para vivir (Ornelas Ramírez \& Ruíz Martínez, 2017).

Hasta el momento no se conocen con precisión las consecuencias que la COVID19 tiene sobre la salud mental de las personas. Hay estudios que apuntan a que las personas con sospechas o diagnóstico confirmado pueden sentir temor a infectarse con 
otro virus que sea fatal (Xiang et al., 2020). Además, la población en general puede experimentar estrés, síntomas de ansiedad y depresión que van de moderados a graves (Wang et al., 2020). Estos síntomas se han identificado con mayor frecuencia e intensidad en el personal sanitario. Para el caso, en un estudio realizado con profesionales de salud en Wuhan, China, se encontraron considerables porcentajes de participantes con síntomas de depresión, ansiedad, insomnio y estrés (Lai et al., 2020).

A esto hay que agregar que, en casos de epidemias por virus parecidos al que produce la COVID-19, hay estudios que identifican el impacto en la salud mental en relación con síntomas de estrés postraumático (posteriores al evento), altos niveles de estrés, ansiedad, ira, sentimientos de estigmatización y temor a contagiar a familiares y colegas, estos últimos dos síntomas especialmente entre personal sanitario (Gardner \& Moallef, 2015). Sin embargo, muchos de los problemas psicológicos que se presentan podrían estar más vinculados con la situación social que se genera y con las medidas para prevenir el contagio del virus (Jeong et al., 2016).

La cuarentena, el aislamiento y el distanciamiento social están entre las principales medidas de salud pública que se toman para evitar o disminuir la exposición a personas que tienen o pueden tener enfermedades contagiosas, como la COVID-19, y así reducir la propagación de diferentes virus (Centers for Disease Control and Prevention, 2020). La cuarentena tiene el objetivo de limitar el movimiento de las personas que han estado en riesgo de exposición a enfermedades contagiosas con el fin de vigilar el potencial desarrollo de la enfermedad y reducir el peligro de propagación. Por otro lado, el aislamiento se refiere a la separación entre personas diagnosticadas con una enfermedad y aquellos casos negativos. Cabe mencionar que es común utilizar ambos términos de manera intercambiable (Brooks et al., 2020). Por otro lado, el distanciamiento social se refiere a las medidas de confinamiento y de mantenerse alejado de otras personas con el fin de evitar el contagio de la COVID-19 (Maragakis, 2020).

A pesar de que estas medidas son efectivas para disminuir los contagios, también tienen repercusiones sociales, económicas y en la salud mental de las personas. Estas repercusiones están vinculadas entre sí y se refuerzan unas a otras. Por ejemplo, una de las consecuencias más severas que ha dejado la pandemia es económica, ya que las personas no pueden continuar con sus actividades laborales y esto impacta en sus ingresos durante y después de la cuarentena. A su vez, esta situación es un factor que aumenta los problemas sociales y el riesgo de padecimientos psicológicos (Brooks et al., 2020). 
En estudios realizados durante la epidemia del Síndrome Respiratorio de Oriente Medio (MERS), en Corea, se reportaron impactos psicológicos negativos debido al aislamiento, relacionados con sensaciones de ansiedad y enojo que persistieron entre $4 \mathrm{y}$ 6 meses después de suspender las medidas (Jeong et al., 2016). Por otra parte, durante la epidemia del Síndrome Respiratorio Agudo Grave, en Canadá se encontró que las personas en cuarentena sufrían niveles significativos de angustia, con síntomas de estrés postraumático y depresión, los cuales resultaban más altos a mayor tiempo de cuarentena, a menor ingreso económico en casa y al contacto con personas contagiadas (Hawryluck et al., 2004).

Sin embargo, aún existe un vacío de información científica sobre los efectos que la COVID-19, la cuarentena y la situación social que se ha generado tienen sobre lo población. Esto es una limitante al momento de crear programas de atención social y psicológica, por tanto, deja en evidencia la necesidad de estudiar este fenómeno desde diferentes áreas del conocimiento (sociológica, psicológica y económica, entre otras) para tener una comprensión holística de su impacto sobre las personas. Tomando en cuenta lo expuesto, el presente estudio tiene el propósito de analizar las preocupaciones vinculadas con la COVID-19, el confinamiento y su relación con la salud mental a partir de las experiencias subjetivas de una muestra de informantes hondureños (entre los que se incluyó personal del área de la salud, empleados del sector formal -trabajando in situ y desde casa- e informal de la economía, desempleados y población general).

\section{Materiales y métodos}

\section{Técnicas de recolección de información e informantes}

El presente estudio tiene enfoque cualitativo, con un diseño narrativo, idóneo para ahondar en las experiencias subjetivas que los informantes tienen sobre la temática de interés (Hernandez Sampieri et al., 2014). Para ello se realizaron entrevistas semiestructuradas que incluyeron preguntas como las siguientes: "Cuénteme, ¿cómo se ha sentido durante la cuarentena?” y “Qué es lo que más le preocupa en este momento? ¿Por qué?"

Entre los informantes estaban a) cuatro empleados del área de salud, b) cuatro participantes sin empleo, c) cuatro empleados del sector informal de la economía, d) cuatro empleados del sector formal que continúan laborando desde casa y e) personas que continúan laborando en su espacio de trabajo pese a la cuarentena (in situ). De esta manera 
se logró un total de 20 entrevistas, 10 participantes eran del sexo femenino y 10 del masculino, con edades entre $\operatorname{los} 22$ y los 60 años. La tabla 1 contiene un resumen demográfico de la muestra.

Tabla 1

Descripción de los participantes del estudio por sexo y edad

\begin{tabular}{ccc}
\hline \multirow{2}{*}{ Agrupación } & \multicolumn{2}{c}{ Sexo } \\
& Masculino & Femenino \\
\hline Trabaja desde casa & 24 y 26 años & 26 y 30 años \\
Trabaja in situ & 25 y 54 años & 28 y 29 años \\
Trabaja en el campo de la salud & 28 y 43 años & 31 y 60 años \\
Empleo informal & 31 y 45 años & 31 y 47 años \\
Sin empleo & 22 y 31 años & 23 y 28 años \\
Total & 10 & 10 \\
\hline
\end{tabular}

Nota. Elaboración propia

Cabe agregar que la selección de informantes se realizó de manera noprobabilística, por conveniencia, según los criterios de inclusión (sexo, edad, voluntariedad y tipo de informante). Las entrevistas se llevaron a cabo de forma virtual, por medio de videollamadas. Previo al inicio de estas sesiones, a los potenciales informantes se les mandó vía correo electrónico un consentimiento informado digital en el cual se detallaba el propósito del estudio, que la participación era voluntaria, sin que mediara ninguna compensación económica, y que la información se manejaría de manera confidencial. Además, se les solicitó permiso de grabar la entrevista y se les dieron los datos de contacto del investigador principal, incluyendo su afiliación institucional. Todo ello de acuerdo con los lineamientos éticos de investigación aprobados por la Maestría en Psicología Clínica de la Universidad Nacional Autónoma de Honduras.

\section{Estrategias de análisis de información}

La información recogida se trabajó mediante una estrategia de análisis temático. Lo primero que se hizo fue identificar las categorías temáticas principales en el discurso de los informantes. Después se procedió a crear subcategorías que evidenciaran distintas ramificaciones conceptuales de los temas principales. Para facilitar este proceso se realizó una codificación descriptiva de las categorías y subcategorías, lo que permitió ordenar más fácilmente el discurso. A partir de este ordenamiento se procedió a crear un texto descriptivo-interpretativo que intentaba reflejar la subjetividad que los informantes transmitieron en las entrevistas (Schettini \& Cortazzo, 2015). Este texto pasó después por 
un proceso de revisión y afinamiento conceptual, hasta convertirse en los resultados que se presentan en este trabajo.

\section{Resultados}

\section{Preocupación por la salud}

Preocupación por la salud propia

Un tema que se repite en el discurso es la preocupación por la salud propia, la mayor parte de los participantes manifiestan temor por el contagio de COVID-19, en tal sentido una de las entrevistadas comenta:

Horrible, porque psicológicamente uno comienza a tener dudas y uno dice " ¿y si soy asintomático y si a pesar de que no tengo síntomas, dicen que el virus tarda no sé cuánto tiempo en incubarse, y si lo tengo?", entonces los nervios, aunque uno no tenga síntomas, se sienten muchísimo. -Mujer, 29 años, trabajo in situ.

Al indagar acerca de la sensación de sentirse enfermos, muchos comentan que han experimentado síntomas físicos vinculados con la COVID-19, pero que ellos mismos son conscientes de que esto es producto de aspectos psicológicos relacionados con el temor al contagio. Ante esto comentan:

Siempre tomo mis precauciones y todas las medidas, gracias a Dios tengo el conocimiento, y cuando llego a casa me quito la ropa, me baño y todo, siempre preocupado. A veces me he sentido como si estuviera enfermo, pero después descubro que es psicológico, a veces siento que me arde la garganta y cosas así y después miro que es psicológico. -Hombre, 28 años, desempleado.

En este mismo sentido, al investigar acerca de las preocupaciones actuales, una informante comenta que uno de sus mayores temores es la reincorporación laboral, dada la actual situación de COVID-19. Al analizar los temas que emergen del discurso de los informantes es evidente el temor que manifiestan a salir del hogar. Esto se evidencia en el siguiente fragmento de entrevista:

Que me manden a trabajar el día de mañana, creo que el riesgo de contagio básicamente. Si no queremos salir, al menos personalmente, es más por contagiarme o afectar a mi familia también y entrar a una situación de enfermedad. No es como que me afecte trabajar (...) el miedo es más por el contagio. - Mujer, 30 años, trabajo remunerado en casa. 
Una de las principales reacciones psicológicas ante la cuarentena ha sido la ansiedad, tanto en relación con la enfermedad como ante el posible contagio y el tiempo de movilización limitado. Una de las informantes comenta al respecto:

Recién iniciada la cuarentena a mí me dio una crisis de ansiedad donde nunca me había dado nada, en la noche comenzó a faltarme la respiración y eso. -Mujer, 47 años, empleo informal.

Preocupación por la salud de terceros

Otro tema emergente es la preocupación que muestran los entrevistados por la salud de sus familiares o de terceros significativos. De manera que se percibe que el contagio propio puede propiciar el contagio de los seres queridos, esto se evidencia en el siguiente fragmento:

En este momento lo que más me preocupa es estar contaminado del virus, pero no por mí, sino que más bien por mi familia, sobre todo por los niños pequeños. Entonces sí, el miedo es contaminar a los demás, contaminarme para contaminar a los demás, ese es el temor. -Hombre, 31 años, trabaja en el campo de la salud.

Un caso particular es la preocupación que los familiares muestran por los empleados de la salud, según narra una informante:

Al inicio fue difícil, ¿por qué?, tengo tres hijos (...) y como que se ponían los tres de acuerdo para hacerme la vida imposible diciéndome de que cómo era posible que yo me iba para la calle, y no pensar en ellos. -Mujer, 60 años, trabaja en el campo de la salud.

\section{Conciencia del contagio de COVID-19}

La preocupación por la salud propia y la de terceros indica que los informantes tienen conciencia manifiesta de la elevada tasa de contagio que tiene la COVID-19. De manera particular, el discurso de los entrevistados evidencia cierto grado de preparación psicológica ante el virus, en donde el contagio comunitario se percibe como inminente. Según los participantes:

Pero yo ya sabía que iba a venir en algún momento. Si vino desde China a Honduras, como no iba a llegar acá, es ilógico. Entonces ya estaba mentalizada y sabida que en cualquier momento. -Mujer, 31 años, desempleada. 
Sin embargo, al no considerarse población vulnerable, hay informantes que devalúan el riesgo de contagio de COVID-19. Sumado a esto, hay quienes perciben que en su comunidad hay personas que se muestran incrédulas respecto a las repercusiones que tiene la enfermedad. Esto no solo se manifiesta por medio de comentarios, sino que también en la falta de acatamiento de las medidas de cuarentena y bioseguridad. Así lo dicen los informantes:

Creo que se ha visto un poco exagerada por medios de comunicación. Creo que no soy una población de riesgo y el que me enferme o no, no me pone en riesgo como una persona diabética o con problemas de circulación. -Mujer, 26 años, trabaja desde casa.

Aquí donde vivo hay mucha gente indiferente, que no le importa la crisis que está generando esto del COVID-19, y la indiferencia. Yo he platicado con algunas personas en la calle, cuando voy a la pulpería, o el mercadito y no creen en esto, dicen que es mentira, que es algo inventado, entonces, y al final ellos corren un riesgo porque pasan en la calle, veo que se reúnen, hacen reuniones, fiestas, he sido testigo de eso. Visitan a sus amigos en las casas, familiares se visitan entre ellos, veo niños en la calle jugando con sus padres, no les importa. -Hombre, 45 años, empleado del sector informal de la economía.

Información sobre la situación de COVID-19

Al indagar respecto al consumo mediático relativo a la situación de COVID-19, muchos entrevistados reportan informarse por redes sociales, cadenas nacionales de radio y televisión, así como por comunicaciones personales con terceros. Adicionalmente, se percibe que exponerse a la información respecto a estadísticas diarias de COVID-19 puede ser perjudicial para la salud mental. Según comentan los informantes:

Actualmente dejé de leer todos los medios, de leer en redes sociales, no miro noticieros. Actualmente digo "no quiero saber nada ya". Es contradictorio porque al inicio me alarmaba muchísimo, pasaba todo el tiempo alarmada, me generaba más preocupación. La verdad me ponía con un estado de ánimo bajísimo (...) yo he optado por no ver nada y es mejor. - Mujer, 29 años, trabaja in situ. 
Preocupación por el ámbito económico

Una de las preocupaciones más recurrentes de los entrevistados corresponde al ámbito económico. En tal sentido, las medidas de cuarentena han creado sentimientos de incertidumbre en la condición laboral de algunos informantes. De manera específica, hay narraciones que denotan que se han cerrado oportunidades de acceso al mercado laboral, ha habido suspensiones de trabajadores o una disminución en el ingreso económico de los participantes, detonando así reacciones de estrés. Un ejemplo de ello se evidencia en el siguiente fragmento:

Debido a la suspensión laboral estoy preocupado por mis finanzas. Antes no se comía tanto como ahora y los gastos de luz, agua, teléfono, pues hay que tenerlos. La colegiatura de mis hijos es lo que más me preocupa y esto me ha bajoneado un poco y me he sentido un poco triste y para decir que estaba preparado económicamente, no lo estaba. -Hombre, 28 años, desempleado.

\section{Alteraciones en los hábitos}

\section{Hábitos alimenticios}

Durante la cuarentena por COVID-19 algunas personas reportan cambios significativos en sus hábitos alimenticios. Por una parte, hay quienes han consumido mayores cantidades de comida debido a la ansiedad que la situación de crisis les provoca. Ante esto, una de ellas comenta:

En cuanto a la alimentación, creo que me he pasado un poco, me ha dado más ansiedad. Sí he comido más, me he descuidado un poco de mi dieta alimenticia, he estado comiendo un poco más, he subido más de peso debido a la ansiedad por todo esto. -Mujer, 47 años, empleo informal.

Lo contrario también se ha reportado. Hay personas que comentan haber disminuido su ingesta de alimentos ya sea porque no sienten tanta hambre -presentan fluctuaciones constantes en cuanto a las cantidades de comida consumidas- o por el limitado acceso a los mismos. En este sentido expresan:

Más restringidos porque ya no podés comer como antes. Tenés que limitarte muchas cosas: una porque no las podés comprar todos los días, tenés que economizar porque te terminás la comida en una semana y no podés salir a comprar. -Mujer, 42 años, trabajo remunerado en casa. 


\section{Hábitos del sueño}

Los hábitos del sueño también han sufrido un impacto negativo a causa de la cuarentena, tanto en la cantidad de horas que las personas logran dormir como en la calidad del sueño y el descanso percibido. Además, el cambio de rutina ha hecho que la higiene del sueño experimente cambios significativos. Sobre esto los entrevistados comentan:

Mal, la verdad me ha afectado mucho, tengo un problema de no poder dormir bien. A veces siento que duermo, pero igual me despierto cansada, sigo pensando aun estando dormida, no lo sé, pero sí siento, pero no he descansado como antes. - Mujer, 29 años, trabajo in situ.

Duermo de día y de noche, generalmente me acuesto a las 5:00 o 6:00 de la mañana porque no puedo dormir, y duermo en el día. -Hombre, 31 años, empleado del sector informal de la economía.

Tiempo de ocio

El tiempo de cuarentena ha significado una limitación para realizar actividades que implican estar en espacios abiertos o fuera de casa. Sin embargo, algunos de los informantes comentan que este tiempo les ha posibilitado practicar actividades de juego que les permiten mantenerse distraídos y fomentar las relaciones familiares, o retomar pasatiempos que por falta de tiempo no podían realizar antes. Dos entrevistados comentan lo siguiente sobre este aspecto:

Particularmente me gusta tocar guitarra, entonces en mis momentos libres me pongo a practicar y, además, me gusta leer de vez en cuando y actualmente estoy leyendo un libro. Esta es la forma en que trato de distraerme cuando tengo tiempo. -Hombre, 31 años, empleado del sector salud.

Las que realiza uno al estar en casa con la familia, hemos visto películas, hemos compartido momentos que hace algún tiempo no hacíamos juntos. -Hombre, 54 años, trabajo in situ.

\section{Discusión}

Los resultados de este estudio revelan que los participantes experimentan preocupaciones por la salud propia y de terceros, lo que indica que están conscientes sobre el peligro de contagio de la COVID-19. Adicionalmente, comentan que la exposición 
constante a la información que brindan los medios de comunicación sobre la COVID-19 es causante de estrés, por lo que han optado por reducir el consumo mediático. Estos resultados son coherentes con un estudio previo en el cual se encontró que el temor al COVID-19 está determinado por la ansiedad por la salud propia, la preocupación por el bienestar de los seres queridos y el uso regular de las redes sociales (Mertens et al., 2020). A pesar de esto, hay personas que por ser jóvenes y tener buena salud consideran que la COVID-19 es una situación de bajo riesgo para ellas. Esta actitud puede detonar la práctica de conductas que implican el descuido de las medidas preventivas de bioseguridad, lo que extensivamente aumentaría el riesgo de contagio entre la población.

Los resultados de la presente investigación son coherentes con estudios internacionales recientes que revelan que el impacto psicológico de una cuarentena puede incluir sintomatología de insomnio, irritabilidad, ansiedad, angustia y sensación de pérdida de la libertad (Piña Ferrer, 2020). Además, las medidas preventivas, como el confinamiento y el distanciamiento social, han tenido repercusiones económicas que se convierten en fuentes adicionales de estrés para los individuos (Caqueo-Urízar et al., 2020). Según reportan los participantes en el presente estudio, estos estresores están vinculados con las preocupaciones por la reducción del acceso al mercado laboral, la suspensión o cancelación de contratos de trabajo y la disminución de los ingresos financieros.

Adicionalmente, los hallazgos revelan alteraciones cualitativas y cuantitativas en los hábitos de sueño y el consumo de alimentos, asociadas con la crisis de la COVID-19. Esto pone de manifiesto que el confinamiento y el temor al contagio del virus podrían dejar entre la población hondureña secuelas que se prolongarán incluso después de que se atempere la situación actual. Para el caso, la alteración de los hábitos de sueño ha sido vinculada con problemas de salud mental como la depresión, irritabilidad, regulación emocional y riesgo suicida (Vilchez-Cornejo et al., 2016). Además, la persistencia de estas alteraciones podría suponer una dificultad práctica cuando las personas tengan que retomar su rutina laboral o estudiantil. Considerando esto, el abordaje de los problemas de sueño es indispensable en tiempos de confinamiento, ya que de esta forma se reduce el impacto del estrés y previene el deterioro en las interacciones sociales de las personas (Altena et al., 2020).

Por otro lado, los entrevistados en el presente estudio reportan variaciones en sus hábitos alimenticios. Esto ha sido evidenciado también en investigaciones realizadas en Italia, que señalan que durante el confinamiento un porcentaje significativo de los 
participantes reportaban un aumento en su peso (Di Renzo et al., 2020). En tal sentido, el desorden en la ingesta de alimentos puede vincularse como causal de sobrepeso, obesidad y desnutrición (Oda-Montecinos et al., 2015). Estos últimos son problemas de salud que pueden impactar sobre la calidad de vida de las personas y, mediante los costos que se generan por atenciones médicas, en los ya debilitados sistemas sanitarias de los países. Además, estudios recientes sugieren que una dieta saludable reduce la susceptibilidad a los efectos a largo plazo de la COVID-19 (Butler \& Barrientos, 2020), por lo que los mismos desórdenes alimenticios que se producen durante el confinamiento podrían incrementar la vulnerabilidad física de las personas al virus.

Los participantes también comentan que a raíz de la situación provocada por la pandemia ahora disponen de tiempo de ocio. Este es un factor protector importante contra el estrés y, además, hay estudios que han encontrado que tiene beneficios importantes para la salud mental (Goodman et al., 2017). El confinamiento también ha dado la oportunidad de realizar actividades con las personas con quienes se cohabita (típicamente familia y/o amistades), promoviendo la cohesión dentro de estos grupos. Es interesante que los participantes en esta investigación valoran positivamente la cohabitación familiar, pues estudios realizados en muchos países han reportado un aumento en los casos de violencia doméstica vinculada con la convivencia en cuarentena. En este sentido, se señala que en familias disfuncionales el confinamiento puede exacerbar los problemas sociales, volviéndose un factor de riesgo para la salud mental y física (Campbell, 2020).

Entre las limitaciones de la presente investigación se encuentra el medio utilizado para realizar las entrevistas, que, al ser virtuales, pueden padecer de un reducido vínculo interpersonal entre informantes y entrevistador. Además, no hay en la muestra ninguna persona que tenga o haya tenido COVID-19, condición que podría aportar interesantes elementos de análisis respecto a la preocupación por el contagio y las consecuencias de la enfermedad. A esto se suma que la propia naturaleza no probabilística del muestreo implica que los hallazgos pueden no ser representativos de la población hondureña.

Sin embargo, incluso con las limitaciones descritas los resultados obtenidos son relevantes y a partir de ellos se pone en evidencia que es necesario que se realicen investigaciones similares enfocadas en poblaciones o procesos específicos como el duelo vinculado con la COVID-19, las experiencias subjetivas de las personas que han sido diagnosticadas positivas, los efectos psicosociales de las medidas de confinamiento y la relación entre confinamiento y violencia de género. También es necesario considerar como objetos de estudio a las poblaciones que son particularmente vulnerable al estrés, 
como aquellos que trabajan en los sistemas de salud, quienes cuidan niños, los adultos mayores, personas con enfermedades de base y sus cuidadores y personas en aislamiento por diversas razones (World Health Organization, 2020). Asimismo, es recomendable realizar réplicas del estudio en diferentes países para cruzar información y tener una visión más amplia del impacto de la situación sobre la salud mental. Se sugiere que futuras investigaciones incluyan diseños mixtos que permitan una comprensión integral del fenómeno.

En conclusión, las personas que participaron en este estudio tienen dos tipos de preocupaciones fundamentales en relación con la situación provocada por la COVID-19. Una es la preocupación por la salud propia y la de las personas cercanas, es decir, el temor a contagiarse y contagiar a los demás. La otra es una preocupación vinculada con la problemática económica que está produciendo el confinamiento en materia de pérdida de empleos y reducción de ingresos. Todo esto genera incertidumbre sobre la dificultad a corto plazo de suplir adecuadamente necesidades básicas como la alimentación y la educación. Adicionalmente, los entrevistados reportan alteraciones en sus hábitos alimenticios y de sueño, siendo este un factor de riesgo para la salud mental. Por último, el tiempo de ocio es un factor protector importante para los informantes. Las implicaciones de estos hallazgos sugieren que la preocupación por la COVID-19 y el confinamiento han detonado reacciones psicológicas importantes que deben ser abordadas desde el ámbito de la salud pública. 


\section{Referencias}

Altena, E., Baglioni, C., Espie, C. A., Ellis, J., Gavriloff, D., Holzinger, B., Schlarb, A., Frase, L., Jernelöv, S., \& Riemann, D. (2020). Dealing with sleep problems during home confinement due to the COVID-19 outbreak: Practical recommendations from a task force of the European CBT-I Academy. Journal of Sleep Research, 29(4), e13052. https://doi.org/10.1111/jsr.13052

American Psychological Association. (2020). Mental health. In APA Dictionary of Psychology. https://dictionary.apa.org/mental-health

Brooks, S. K., Webster, R. K., Smith, L. E., Woodland, L., Wessely, S., Greenberg, N., \& Rubin, G. J. (2020). The psychological impact of quarantine and how to reduce it: rapid review of the evidence. The Lancet, 395(10227), 912-920. https://doi.org/10.1016/S0140-6736(20)30460-8

Butler, M. J., \& Barrientos, R. M. (2020). The impact of nutrition on COVID-19 susceptibility and long-term consequences. Brain, Behavior, and Immunity, 87, 53-54. https://doi.org/https://doi.org/10.1016/j.bbi.2020.04.040

Campbell, A. M. (2020). An increasing risk of family violence during the Covid-19 pandemic: Strengthening community collaborations to save lives. Forensic Science International: Reports, 2, 100089. https://doi.org/https://doi.org/10.1016/j.fsir.2020.100089

Caqueo-Urízar, A., Urzúa, A., Aragón-Caqueo, D., Charles, C. H., El-Khatib, Z., Otu, A., \& Yaya, S. (2020). Mental health and the COVID-19 pandemic in Chile. Psychological Trauma: Theory, Research, Practice and Policy. https://doi.org/10.1037/tra0000753

Centers for Disease Control and Prevention. (2020). About Quarantine and Isolation| Quarantine |CDC. https://www.cdc.gov/quarantine/quarantineisolation.html

Despacho de Comunicaciones y Estrategia Presidencial. (2020, May). COVID-19 Honduras: Coronavirus en Honduras. https://covid19honduras.org/

Di Renzo, L., Gualtieri, P., Pivari, F., Soldati, L., Attinà, A., Cinelli, G., Leggeri, C., Caparello, G., Barrea, L., Scerbo, F., Esposito, E., \& De Lorenzo, A. (2020). Eating habits and lifestyle changes during COVID-19 lockdown: an Italian survey. Journal of Translational Medicine, 18(1), 229. https://doi.org/10.1186/s12967-020-02399-5

Gardner, P. J., \& Moallef, P. (2015). Psychological impact on SARS survivors: Critical review of the English language literature. Canadian Psychology/Psychologie Canadienne, 56(1), 123-135. https://doi.org/10.1037/a0037973

Goodman, W. K., Geiger, A. M., \& Wolf, J. M. (2017). Leisure activities are linked to mental health benefits by providing time structure: comparing employed, unemployed and homemakers. Journal of Epidemiology and Community Health, 71(1), 4 LP - 11. https://doi.org/10.1136/jech-2016-207260

Hawryluck, L., Gold, W. L., Robinson, S., Pogorski, S., Galea, S., \& Styra, R. (2004). SARS control and psychological effects of quarantine, Toronto, Canada. Emerging Infectious Diseases, 10(7), 1206-1212. https://doi.org/10.3201/eid1007.030703

Hernandez Sampieri, R., Fernandez Collado, C., \& Baptista, M. del P. (2014). Metodologia de la Investigacion. Mc Graw Hill.

Jeong, H., Yim, H. W., Song, Y.-J., Ki, M., Min, J.-A., Cho, J., \& Chae, J.-H. (2016). Mental health status of people isolated due to Middle East 
Respiratory Syndrome. Epidemiology and Health, 38, e2016048. https://doi.org/10.4178/epih.e2016048

Johns Hopkins University and Medicine. (2020). COVID-19 Map - Johns Hopkins Coronavirus Resource Center. In John Hopkins Coronavirus Resource Center (p. 1). https://coronavirus.jhu.edu/map.html

Lai, J., Ma, S., Wang, Y., Cai, Z., Hu, J., Wei, N., Wu, J., Du, H., Chen, T., Li, R., Tan, H., Kang, L., Yao, L., Huang, M., Wang, H., Wang, G., Liu, Z., \& Hu, S. (2020). Factors Associated With Mental Health Outcomes Among Health Care Workers Exposed to Coronavirus Disease 2019. JAMA Network Open, 3(3), e203976. https://doi.org/10.1001/jamanetworkopen.2020.3976

Maragakis, L. L. (2020). Coronavirus, Social and Physical Distancing and SelfQuarantine |Johns Hopkins Medicine. Johns Hopkins Medicine, 4-7. https://www.hopkinsmedicine.org/health/conditions-anddiseases/coronavirus/coronavirus-social-distancing-and-self-quarantine

Mertens, G., Gerritsen, L., Duijndam, S., Salemink, E., \& Engelhard, I. M. (2020). Fear of the coronavirus (COVID-19): Predictors in an online study conducted in March 2020. Journal of Anxiety Disorders, 74, 102258. https://doi.org/https://doi.org/10.1016/j.janxdis.2020.102258

Oda-Montecinos, C., Saldaña, C., \& Andrés Valle, A. (2015). Comportamientos alimentarios cotidianos y anómalos en una muestra comunitaria de adultos chilenos . Nutrición Hospitaliaria, 32(2), 590-599. https://doi.org/10.3305/nh.2015.32.2.9171

Ornelas Ramírez, A., \& Ruíz Martínez, A. (2017). Salud mental y calidad de vida: Su relación en los grupos etarios. PSIENCIA: Revista Latinoamericana de Ciencia Psicológica, 9. https://doi.org/10.5872/psiencia/9.2.21

Piña Ferrer, L. (2020). El COVID 19: Impacto psicológico en los seres humanos. Revista Arbitrada Interdisciplinaria de Ciencias de La Salud. Salud y Vida, 4(7), 188-199. https://doi.org/10.35381/s.v.v4i7.670

Rodríguez Cahill, C. (2020). Cinco retos psicológicos de la crisis del COVID-19. Journal of Negative \& No Positive Results, 5(6), 569-668. https://doi.org/10.19230/jonnpr.3662

Schettini, P., \& Cortazzo, I. (2015). Análisis de datos cualitativos en la investigación social: Procedimientos y herramientas para la interpretación de información cualitativa. Universidad de La Plata. http://sedici.unlp.edu.ar/bitstream/handle/10915/49017/Documento_completo .pdf? sequence $=1$

Shereen, M. A., Khan, S., Kazmi, A., Bashir, N., \& Siddique, R. (2020). COVID-19 infection: Origin, transmission, and characteristics of human coronaviruses. Journal of Advanced Research, 24, 91-98. https://doi.org/10.1016/j.jare.2020.03.005

Sosa, E. (2020, June 19). Honduras: del colapso sanitario al colapso político | CESPAD. https://cespad.org.hn/2020/06/19/analisis-honduras-del-colapsosanitario-al-colapso-politico/

Vilchez-Cornejo, C., Torres-Román, S., Tereza, P., la Cruz, C.-D., Jesús, J., \& Alberto, L. (2016). Salud mental y calidad de sueño en estudiantes de ocho facultades de medicina humana del Perú. Revista Chilena de Neuropsiquiatría, 54(4), 272-281. https://www.redalyc.org/pdf/3315/331549488002.pdf

Wang, C., Pan, R., Wan, X., Tan, Y., Xu, L., Ho, C. S., \& Ho, R. C. (2020). Immediate Psychological Responses and Associated Factors during the Initial 
Stage of the 2019 Coronavirus Disease (COVID-19) Epidemic among the General Population in China. International Journal of Environmental Research and Public Health, 17(5), 1729. https://doi.org/10.3390/ijerph17051729

World Health Organization. (2020). Mental health and psychosocial considerations during the COVID-19 outbreak. https://www.who.int/docs/defaultsource/coronaviruse/mental-health-considerations.pdf?sfvrsn=6d3578af 2

Wu, Z., \& McGoogan, J. M. (2020). Characteristics of and Important Lessons from the Coronavirus Disease 2019 (COVID-19) Outbreak in China: Summary of a Report of 72314 Cases from the Chinese Center for Disease Control and Prevention. In JAMA - Journal of the American Medical Association, 323(13), 1239-1242). https://doi.org/10.1001/jama.2020.2648

Xiang, Y.-T., Yang, Y., Li, W., Zhang, L., Zhang, Q., Cheung, T., \& Ng, C. H. (2020). Timely mental health care for the 2019 novel coronavirus outbreak is urgently needed. The Lancet Psychiatry, 7(3), 228-229. https://doi.org/10.1016/S2215-0366(20)30046-8 